\title{
Comparação da prescrição de antibacterianos em 2001 e 2007: um estudo na rede Médicos Sentinela
}

Eleonora Paixão, * Maria João Branco, ** Emanuel Rodrigues, *** José Marinho Falcão****

\section{RESUMO}

Objectivos: Comparar a prescrição de cefalosporinas e quinolonas pelos médicos de Medicina Geral e Familiar (MGF), participantes da Rede Médicos-Sentinela (MS), em 2007, com a observada num estudo semelhante realizado em 2001, estimando: i) o número de antibacterianos (AB) prescritos por 1.000 utentes; ii) a percentagem de prescrições de cefalosporinas; e iii) a percentagem de prescrições de quinolonas.

Tipo de estudo: Observacional, descritivo, transversal.

Local: Centros de Saúde de Portugal onde trabalham os médicos da Rede MS.

População: Lista de utentes dos médicos da Rede MS.

Métodos: $O$ estudo foi realizado no âmbito da Rede MS que permite estimar indicadores de base populacionais. A notificação das prescrições de $A B$ decorreu em 2001 e em 2007. Foram calculados índices de frequência anual da prescrição de $A B$ (nANTI = número de prescrições de $A B$ por 1.000 indivíduos), desagregados por sexo e idade.

Resultados: Foram estudadas 12.184 prescrições de AB em 2001 e 9.034 em 2007, relevando-se: o número de prescrições de cefalosporinas por 1.000 utentes, assim como a percentagem de prescrição no total de prescrições de $A B$, foram menores em 2007 relativamente a 2001 (respectivamente, nANTI = 8,2 e 10,1\%, em 2007 e nANTI = 11,1 e 11,9\%, em 2001); o número de prescrições de quinolonas por 1.000 utentes foi menor em 2007 relativamente a 2001 (respectivamente, nANTI = 13,2 e nAN$\mathrm{TI}=14,2)$ mas a percentagem de prescrição no total de prescrições de AB foi maior em 2007 do que em 2001 (respectivamente, $16,2$ e $15,3 \%)$.

Conclusões: O número de prescrições de cefalosporinas e de quinolonas por 1.000 utentes foi menor em 2007 relativamente a 2001. De 2001 para 2007, a percentagem de prescrição foi menor nas cefalosporinas mas superior nas quinolonas. $O$ grupo etário dos 75 e mais anos registou um aumento em 2007, quer nas cefalosporinas, quer nas quinolonas, sendo este aumento estatisticamente significativo neste último subgrupo.

Palavras-chave: Antibacterianos; Prescrição; Terapêutica; Médicos de Família; Rede Médicos-Sentinela.

\section{INTRODUÇÃO}

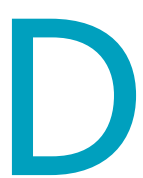
e entre os estados membros da União Europeia/Área Económica Europeia, Portugal, em 2007, posicionou-se em oitavo lugar relativamente ao consumo total de antibacterianos (AB) em ambulatório, com pouco mais de 20 doses diárias definidas/1.000 habitantes/dia. Apesar da melhoria da situação, passados 10 anos (em 1997, 28,8 doses diárias definidas/1.000 habitantes/dia), constatou-se ainda a manutenção de um uso relevante de quinolonas, relativamente aos outros países. ${ }^{1}$
Em Portugal, esta temática faz parte da agenda dos decisores. Com efeito, no Plano Nacional de Saúde 2004-2010, o consumo de AB constituiu uma prioridade na definição de metas para 2010, traduzidas por dois

\footnotetext{
* Estatista, Departamento de Epidemiologia do Instituto Nacional de Saúde Dr. Ricardo Jorge

**Médica de Saúde Pública, Departamento de Epidemiologia do Instituto Nacional de Saúde Dr. Ricardo Jorge

***Estatista, Departamento de Epidemiologia do Instituto Nacional de Saúde Dr. Ricardo Jorge

****Médico de Saúde Pública, Epidemiologista, Departamento de Epidemiologia do Instituto Nacional de Saúde Dr. Ricardo Jorge
} 
indicadores, nomeadamente, «a percentagem de consumo de cefalosporinas no consumo total de $A B$ em regime ambulatório» $\mathrm{e}$ "a percentagem de consumo de quinolonas no consumo total de $A B$ em regime ambulatório».2

A informação disponível permite constatar uma evolução positiva destes indicadores, no sentido de uma maior racionalidade na utilização daqueles $\mathrm{AB}$, constatando-se mesmo que, para as cefalosporinas, a meta proposta para 2010 , de $10 \%$, foi ultrapassada em 2008 , situando-se a sua percentagem de consumo em 9,4\%. A evolução da utilização de quinolonas, ainda que positiva, não se revelou tão favorável, ${ }^{3}$ a meta proposta para 2010 foi de $10,6 \%$, sendo a percentagem de consumo em 2008 de 12,7\%.

A reforçar a pertinência da problemática em estudo, valerá a pena referir alguns dados relativos às vendas deste tipo de medicamentos, em ambulatório, no mercado nacional: No ano 2003, ${ }^{4}$ uma das substâncias activas do grupo das quinolonas ocupou o $20 .^{\circ}$ lugar na tabela das 100 substâncias activas com maior encargo para o Serviço Nacional de Saúde (SNS), tendo em $2007^{5}$ descido para o $31 .^{\circ}$ lugar na tabela. Em relação às cefalosporinas, em 2003, ${ }^{4}$ uma das substâncias activas deste grupo, ocupava o $26 .^{\circ}$ lugar tendo em $2007^{5}$ passado a ocupar a $71 .{ }^{a}$ posição.

A importância global da prescrição deste grupo de fármacos fora do SNS não é facilmente quantificável. Poder-se-á, contudo, admitir que o padrão de prescrição não seja muito diferente no âmbito da Medicina privada e dos vários subsistemas de saúde.

Mais especificamente, a utilização de $\mathrm{AB}$ em Medicina Geral e Familiar (MGF) tem sido, universalmente, objecto de estudo e de reflexão aprofundada. ${ }^{6-8}$

Em Portugal, num estudo ${ }^{9}$ realizado em 2000, no serviço de atendimento complementar na Unidade de Saúde de Vialonga, verificou-se uma elevada prescrição de $\mathrm{AB}$ (23,3\% das 1.722 fichas estudadas). No âmbito deste atendimento, as cefalosporinas corresponderam a 12,9\% do total das prescrições antibacterianas e as quinolonas a 7,7\%.Ainda em 2000, num estudo ${ }^{10}$ de base populacional sobre usos e maus usos dos $\mathrm{AB}$, os resultados apontam para um consumo das cefalosporinas de 18,8\% e das quinolonas de 9,5\%, ocupando respectivamente a terceira e quarta posição dos grupos $\mathrm{AB}$ mais frequentemente dispensados nas farmácias portuguesas.
No ano seguinte, em 2001, a prescrição de AB foi objecto de notificação no âmbito da Rede Médicos-Sentinela (MS). ${ }^{11}$ Em 2007, voltou a existir notificação sobre este tema.

Sendo certo que, em Portugal, era já apreciável o conhecimento sobre prescrição de AB em MGF, considerou-se pertinente realizar um estudo cujo objectivo fosse comparar o padrão de prescrição de cefalosporinas e quinolonas em 2007 com o observado no estudo semelhante realizado em 2001, ${ }^{11}$ na Rede MS. Nomeadamente, pretendeu-se obter estimativas, por sexo e idade, para os seguintes indicadores:

- Número total de prescrições de AB por 1.000 utentes;

- Número de prescrições de AB do grupo das cefalosporinas por 1.000 utentes;

- Percentagem de prescrições de AB do grupo das cefalosporinas no total de $\mathrm{AB}$ prescritos;

- Número de prescrições de $\mathrm{AB}$ do grupo das quinolonas por 1.000 utentes;

- Percentagem de prescrições de AB do grupo das quinolonas no total de $\mathrm{AB}$ prescritos.

\section{MÉTODOS}

\section{Descrição do estudo}

O estudo foi realizado no âmbito da Rede MS. MS é uma rede de médicos especialistas em MGF, cuja sua actividade profissional é desenvolvida em Centros de Saúde do SNS e que, voluntariamente, notificam casos ou episódios de doença e de outras situações relacionadas com saúde dos indivíduos inscritos nas respectivas listas de utentes. Esta notificação é feita semanalmente. A base populacional deste sistema permite calcular estimativas de incidência de várias doenças, bem como estimar índices de frequência de situações relacionadas, como utilização de exames complementares de diagnóstico ou prescrição de medicamentos. ${ }^{12}$ No ano de 2001 estavam inscritos na Rede MS 161 médicos e em 2007 estavam 144 médicos, colocados nos 18 distritos do Continente e nas Regiões Autónomas dos Açores e da Madeira.

\section{Prescrição de antibacterianos}

Foram elegíveis para o estudo todos os medicamentos prescritos do subgrupo farmacoterapêutico dos AB. Contudo, para prossecução dos objectivos deste estudo a análise incidiu sobre as cefalosporinas e quinolo- 
nas ${ }^{13}$ de utilização sistémica, por serem indicadores nacionais da prescrição antibacteriana, conforme referido atrás.

Referenciando a classificação ATC (Anatomical Therapeutic Chemical Code), ${ }^{14}$ foram considerados os $\mathrm{AB}$ de uso sistémico (J01) dos subgrupos: outros AB $\beta$-lactâmicos $(\mathrm{JO1D})=$ cefalosporinas; e quinolonas $(\mathrm{JO1M})=$ quinolonas.

Aos médicos da rede foi solicitado que notificassem todos os $\mathrm{AB}$ que prescreveram a indivíduos pertencentes à sua lista de utentes, durante o ano de 2001 e 2007. Destes, eram elegíveis para notificação não só os $\mathrm{AB}$ prescritos por iniciativa do próprio médico mas, também, os que foram prescritos por solicitação de outro médico, ou mesmo do próprio utente.

\section{Notificação}

O tema «Prescrição de $A B$ », entre outros, fez parte do programa de notificação contínua de MS, em 2001 e em 2007. Foi, por isso, incluída no respectivo boletim de notificação, estando sujeita a notificação semanal. Em 2001, 136 MS participaram e notificaram este tema e em 2007120 médicos realizaram esta notificação.

Colheram-se dados relativos às seguintes variáveis: caracterização dos inquiridos (sexo e idade - consideraram-se para efeitos de análise os grupos etários 0-4; $5-9$; 10-14; 15-24; 25-34; 35-44; 45-54; 55-64; 65-74 e 75 e mais anos); e caracterização da prescrição de $\mathrm{AB}(\mathrm{AB}$ prescrito).

\section{Verificação, codificação, registo informático e validação dos dados}

As notificações referentes à prescrição de $\mathrm{AB}$ foram objecto de uma revisão que incidiu sobre a exaustividade do preenchimento, a legibilidade das respostas não pré-codificadas e a existência de erros. Quando foi identificada qualquer anomalia, a sua correcção, sempre que possível, foi concretizada através de contacto telefónico com o médico participante.

Os dados foram sujeitos a codificação que esteve a cargo de dois codificadores com formação prévia sobre o processo de classificação dos fármacos.

Os dados colhidos foram registados em suporte informático, tendo a base de dados sido submetida a um processo de validação da congruência para identificação de valores impossíveis e de inconsistência entre va- lores ou códigos de variáveis. Os erros encontrados foram rectificados, sempre que tal foi possível, ou transformados em valores em falta.

\section{ANÁLISE DOS DADOS}

A análise de dados centrou-se fundamentalmente em calcular: i) a frequência da prescrição total de $\mathrm{AB}$, desagregada por sexo e idade dos utentes; ii) a frequência da prescrição de $\mathrm{AB}$, distribuída pelos dois subgrupos em estudo; iii) a frequência da prescrição dos subgrupos de $\mathrm{AB}$ desagregada por sexo e idade dos utentes.

A frequência foi estimada através de um índice de base populacional (nANTI $=$ número de antibacterianos/1.000 utentes), aplicado tanto à totalidade da amostra como aos seus estratos. O numerador de nANTI foi constituído pelo número de prescrições de AB, total e desagregado por sexo e grupos etários. O denominador foi constituído pelo «número de pessoas sob observação efectiva». O número de pessoas sob observação efectiva de cada período de tempo varia com o número de médicos que estão em actividade nesse período (semana, total do ano). O número de pessoas sob observação efectiva de uma dada semana obtém-se pelo somatório das listas de utentes dos médicos activos nessa semana, isto é, que nesse período enviaram pelo menos uma notificação ou declararam, expressamente, não terem casos a notificar. Consequentemente, o número de pessoas sob observação efectiva de um dado ano é a média dos valores nas 52 semanas do ano.

A comparação do número de $\mathrm{AB}$ prescritos (por 1.000 utentes), no total e desagregado entre os dois anos, foi feita utilizando o teste de Qui-Quadrado de Pearson. Foi estabelecido em $5 \%$ o nível de significância do teste, tendo-se rejeitado a hipótese nula quando a probabilidade de significância do teste ( $p$-value) foi inferior a este valor, considerando-se, assim, estatisticamente significativas as diferenças observadas. Todos os cálculos foram feitos usando o programa estatístico PASW Statistics 18.0. ${ }^{15}$

\section{RESULTADOS}

Considerou-se pertinente apresentar algumas características dos dois estudos de $2001^{11}$ e de 2007 para comparar a evolução da prescrição de $\mathrm{AB}$ na rede MS. Assim, conforme descrito no quadro I, pode-se observar que em 2001 a rede MS era constituída por um número de médicos superior ao de 2007. Da mesma for- 
QUADRO I. Número de médicos inscritos e que notificaram a prescrição de antibacterianos, de médicos por semana de participação, de consultas, de população utente sob observação, de episódios de doenças com prescrição de antibacterianos e de antibacterianos prescritos, na Rede Médicos-Sentinela em 2001 e em 2007.

\begin{tabular}{lrrr} 
& \multicolumn{1}{c}{2001} & \multicolumn{1}{c}{2007} \\
\hline Médicos inscritos na Rede Médicos-Sentinela & 161 & 144 \\
\hline Médicos que notificaram prescrição AB & 136 & 120 \\
\hline Médicos por semana de participação & 4.308 & 3.381 \\
\hline Utentes sob observação & 130.780 & 111.129 \\
\hline Consultas com prescrição de AB & 11.981 & 8.878 \\
\hline Total de AB prescritos & 12.184 & 9.034 \\
\hline Episódios com prescrição de dois AB & 202 & 156 \\
\hline
\end{tabular}

$\mathrm{AB}$ - antibacterianos

ma, o número de médicos que realizaram notificações sobre a prescrição de $\mathrm{AB}$ também foi em 2007 inferior ao de 2001. Os restantes indicadores (número de utentes sob observação, número de consultas com prescrição de $\mathrm{AB}$, número total de $\mathrm{AB}$ prescritos e número de episódios com prescrição de dois $\mathrm{AB}$ ) foram também superiores em 2001 relativamente a 2007.
No conjunto de todos os $\mathrm{AB}$, o número de prescrições de $\mathrm{AB}$ diminuiu entre $2001 \mathrm{e}$ 2007, de 93,2 para 81,3 prescrições por 1.000 utentes (Quadro II).

Esta diminuição de prescrição ocorreu em todos os grupos etários entre os 0 e 64 anos. No grupo etário mais idoso (75 e mais anos) verificou-se, pelo contrário, um acréscimo relevante, traduzido num aumento do nANTI de 99,8 para 121,5 (Quadro II).

Focando a análise comparativa na utilização dos dois grupos de $\mathrm{AB}$ adoptados como indicadores de consumo deste grupo de fármacos, a saber, as cefalosporinas e as quinolonas, constatou-se um decréscimo global do número de prescrições de cefalosporinas por 1.000 utentes entre 2001 $($ nANTI $=11,1)$ e 2007 (nANTI = 8,2, Quadro III). Esta diminuição verificou-se em ambos os sexos, não se revelando, contudo, estatisticamente significativa (Quadro III). Relativamente à idade, as diminuições verificadas revelaram-se significativas nos grupos etários 0-4 ( $p<0,001), 10-14$ ( $p=0,006), 15-24$ ( $\underline{p}<0,001)$, 25-34 ( $p=0,001), 35-44(p=0,031), 45-54(p=0,043) \mathrm{e}$ $55-64(p=0,011)$. No grupo etário de 75 e mais anos, no

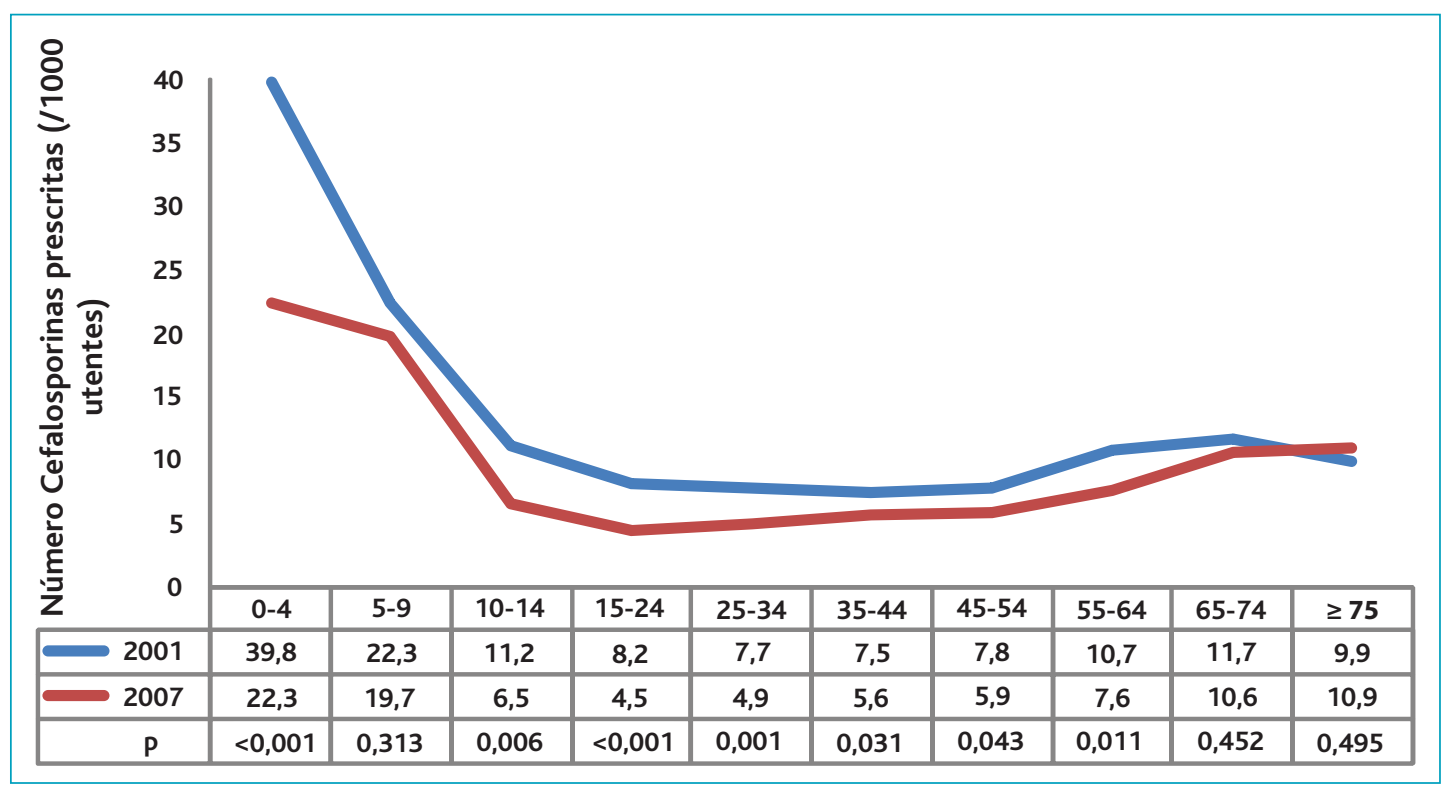

Figura 1. Número de prescrições de cefalosporinas (por 1.000 utentes) em 2001 e 2007, por grupo etário. 


\begin{tabular}{|c|c|c|}
\hline \multicolumn{3}{|c|}{$\begin{array}{l}\text { QUADRO II. Número de prescrições de antibacterianos } \\
\text { (por } 1.000 \text { utentes e número total de prescrições), no } \\
\text { total e por grupo etário, em } 2001 \text { e } 2007 \text {. }\end{array}$} \\
\hline & 2001 & 2007 \\
\hline $\begin{array}{l}\text { Prescrições de } A B / 1.000 \text { utentes } \\
\text { (total de prescrições) }\end{array}$ & $\begin{array}{r}93,2 \\
(12.184)\end{array}$ & $\begin{array}{r}81,3 \\
(9.034)\end{array}$ \\
\hline $\begin{array}{l}\text { Idade } \\
0-4\end{array}$ & $\begin{array}{r}233,4 \\
(1.465)\end{array}$ & $\begin{array}{l}143,0 \\
(841)\end{array}$ \\
\hline $5-9$ & $\begin{array}{l}135,9 \\
(906)\end{array}$ & $\begin{array}{l}117,1 \\
(689)\end{array}$ \\
\hline $10-14$ & $\begin{array}{r}86,4 \\
(617) \\
\end{array}$ & $\begin{array}{r}63,1 \\
(360) \\
\end{array}$ \\
\hline $15-24$ & $\begin{array}{r}79,1 \\
(1.421) \\
\end{array}$ & $\begin{array}{r}61,1 \\
(793) \\
\end{array}$ \\
\hline $25-34$ & $\begin{array}{r}73,8 \\
(1.155) \\
\end{array}$ & $\begin{array}{r}57,0 \\
(996)\end{array}$ \\
\hline $35-44$ & $\begin{array}{r}72,4 \\
(1.368) \\
\end{array}$ & $\begin{array}{r}62,3 \\
(1.025) \\
\end{array}$ \\
\hline $45-54$ & $\begin{array}{r}75,3 \\
(1.155)\end{array}$ & $\begin{array}{r}70,0 \\
(1.024)\end{array}$ \\
\hline $55-64$ & $\begin{array}{r}92,4 \\
(1.249) \\
\end{array}$ & $\begin{array}{r}89,8 \\
(1.080) \\
\end{array}$ \\
\hline $65-74$ & $\begin{array}{r}103,7 \\
(1.404)\end{array}$ & $\begin{array}{r}101,3 \\
(1.085)\end{array}$ \\
\hline 75 e mais anos & $\begin{array}{r}99,8 \\
(1.070)\end{array}$ & $\begin{array}{r}121,5 \\
(1.141)\end{array}$ \\
\hline
\end{tabular}

$A B$ antibacterianos

único grupo em que se observou um acréscimo, este não foi significativo ( $p=0,495$, Figura 1). Em 2007, neste grupo, as cefalosporinas de terceira geração representaram $26,7 \%$ das prescrições. Este subgrupo de $A B$ correspondeu a $11,9 \%$ da totalidade de $\mathrm{AB}$ prescritos em 2001, descendo para 10,1\% em 2007 (Quadro IV).

As prescrições de quinolonas por 1.000 utentes diminuíram entre $2001($ nANTI $=14,2)$ e $2007($ nANTI = 13,2, Quadro III). Também neste grupo a diminuição da prescrição verificada em ambos os sexos não se revelou estatisticamente significativa (Quadro III). Em todos os grupos etários com menos de 75 anos, pode-se constatar a diminuição da prescrição do ano de 2001 para o ano de 2007 (Figura 2). Os grupos etários que re-

\begin{tabular}{|c|c|c|c|}
\hline \multicolumn{4}{|c|}{$\begin{array}{l}\text { QUADRO III. Número de antibacterianos prescritos } \\
\text { (por } 1.000 \text { utentes), no total e por sexo, segundo o } \\
\text { grupo ATC (Anatomical Therapeutic Chemical Code) }{ }^{14} \\
\text { das cefalosporinas e quinolonas, em } 2001 \text { e em } 2007 .\end{array}$} \\
\hline & 2001 & 2007 & $\mathrm{p}^{* *}$ \\
\hline Cefalosporinas & 11,1 & 8,2 & $<0,001$ \\
\hline Sexo & & & 0,927 \\
\hline Masculino & 9,3 & 7,1 & \\
\hline Feminino & 12,8 & 9,2 & \\
\hline $\mathrm{p}^{*}$ & $<0,001$ & $<0,001$ & \\
\hline Quinolonas & 14,2 & 13,2 & 0,031 \\
\hline Sexo & & & 0,876 \\
\hline Masculino & 9,2 & 9,1 & \\
\hline Feminino & 18,8 & 17,0 & \\
\hline $\mathrm{p}^{*}$ & $<0,001$ & $<0,001$ & \\
\hline
\end{tabular}

$P^{*}$-refere-se à comparação entre as classes da variável - teste de QuiQuadrado de Pearson.

$P^{* *}$ - refere-se à comparação entre os dois anos - teste de Qui-

Quadrado de Pearson.

velaram diferenças estatisticamente significativas foram: dos 15-24 $(p<0,001)$ e dos 25-34 $(p=0,003)$. Apenas se registou um acréscimo da prescrição de quinolonas por 1.000 utentes no grupo etário com 75 e mais anos de idade (nANTI = 26,8 em 2001 enANTI $=34,5$ em 2007), sendo este aumento estatisticamente significativo ( $p=0,002$, Figura 2 ). O subgrupo das quinolonas representou 15,3\% dos AB prescritos em 2001 e 16,2\% em 2007 (Quadro IV).

\section{DISCUSSÃO}

Este estudo pretendeu comparar a prescrição de $\mathrm{AB}$ em MGF, nos anos de 2001 e 2007. Em ambos os anos a metodologia utilizada foi semelhante, tendo-se utilizado a rede de MS e incluído o tema em estudo no programa de notificação contínua.

Através da notificação semanal, obteve-se uma grande massa de dados, que permitiu uma análise bastante detalhada, apesar de aqui só se apresentarem os resultados que se consideraram mais pertinentes. Contudo, os padrões encontrados podem não corresponder absolutamente aos que se verificam no conjunto da 
QUADRO IV. Comparação da percentagem de prescrições na Rede de Médicos-Sentinela e do consumo em ambulatório no Continente de antibacterianos (cefalosporinas e quinolonas) em 2001, 2002 e 2007.

\begin{tabular}{|l|c|c|c|l|c|} 
& 2001 & 2002 & \multicolumn{2}{|c|}{2007} & $\begin{array}{c}\text { Meta em } \\
2010\end{array}$ \\
\hline & MS* & Continente** $^{*}$ & MS* & Continente** & \\
\hline Cefalosporinas (\%) & 11,9 & 12,6 & 10,1 & 10,5 & 10,0 \\
\hline Quinolonas (\%) & 15,3 & 14,9 & 16,2 & 13,5 & 10,6 \\
\hline
\end{tabular}

MS Rede Médicos Sentinela

* valor percentual de prescrição no total de antibacterianos prescritos, no âmbito da Rede Médicos Sentinela

** valor percentual de consumo no total de antibacterianos consumidos, em ambulatório, no Continente

prescrição em MGF, no país, nomeadamente a sua evolução no tempo.

Quatro potenciais viéses podem contribuir para essa eventual discrepância. Em primeiro lugar, relativamente à representatividade, a população sob observação, assim como a amostra de médicos, não foi seleccionada como uma amostra aleatória da população portuguesa. A utilização de uma amostra de conveniência, constituída por médicos de MGF que trabalham no SNS e que participam voluntariamente na rede
MS, visa possibilitar uma elevada notificação de casos e promover uma boa qualidade da informação. Contudo, certos grupos profissionais (por exemplo, funcionários públicos, empregados bancários) e a população em estratos economicamente mais elevados poderão estar sub-representados, uma vez que podem recorrer a subsistemas de saúde ou a seguros de saúde privados em vez da utilização de Centros de Saúde. Em segundo lugar, os Médicos de Família que pertencem à rede MS podem ter padrões de prescrição diferentes dos colegas de carreira que não integram a rede MS. Em terceiro lugar, cada médico participante poderá ter falhado a notificação de uma percentagem da totalidade das prescrições que realizou. Como a decisão, ou a oportunidade, de notificar poderão não ser independentes do $\mathrm{AB}$ prescrito, existe um potencial viés sobretudo se aquela percentagem tiver sido elevada, o que é impossível de estimar. Finalmente, a circunstância de os médicos saberem que estão a notificar para fins de um estudo poderá, mesmo que de forma não intencional, ter alterado o seu padrão habitual de pres-

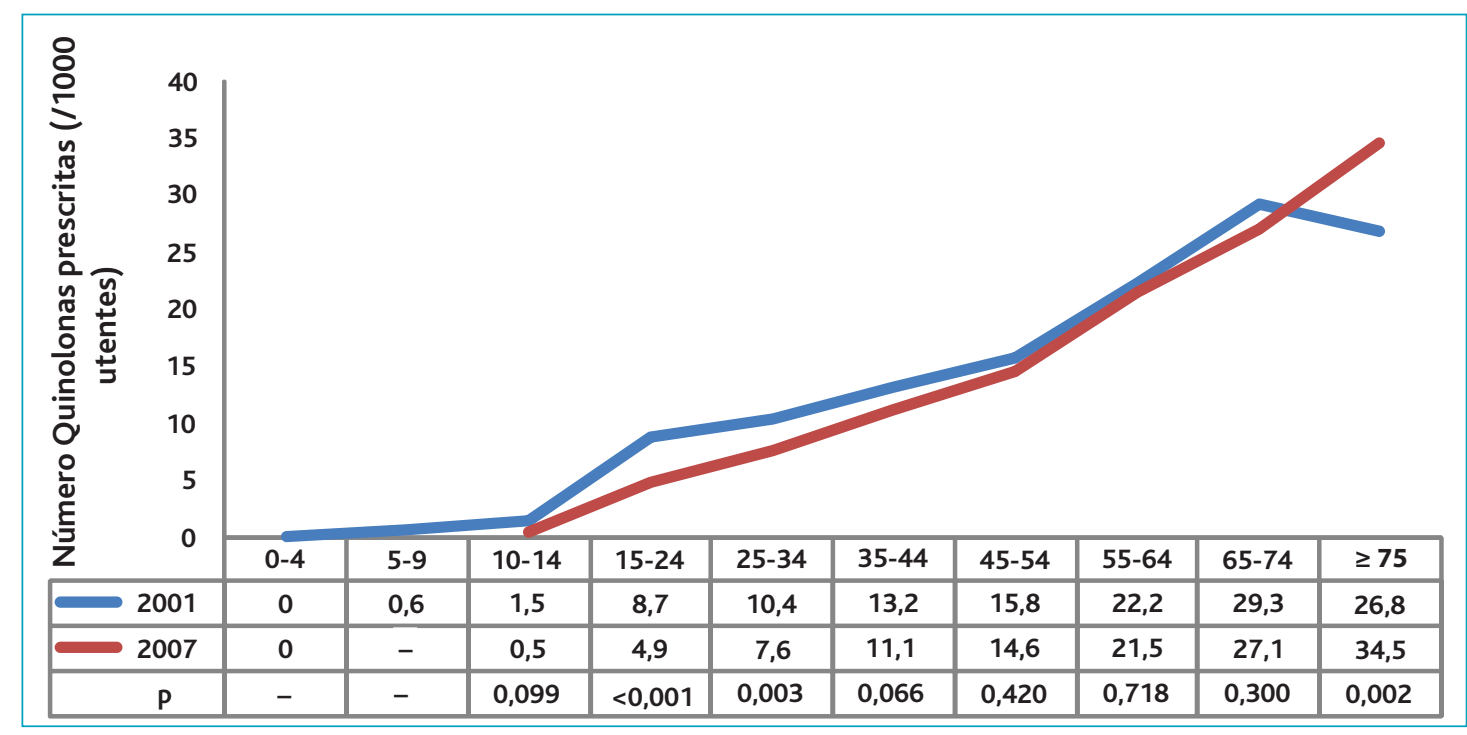

Figura 2. Número de prescrições de quinolonas (por 1.000 utentes) em 2001 e 2007, por grupo etário. 
crição. Contudo, presume-se que a longa duração do período de notificação tenha criado muito precocemente um quadro de rotina que terá atenuado modificações substanciais na prescrição. Nas circunstâncias descritas, e não permitindo os dados a discussão com mais fundamento sobre a existência, o sentido e a dimensão de eventual viés de selecção e de notificação, a interpretação dos resultados deve ter em conta o exposto.

O valor do índice nANTI (número de antibacterianos prescritos/1.000 utentes) pode também sofrer algum viés com origem no seu denominador (número de pessoas sob observação efectiva). Como foi descrito, o número de pessoas sob observação efectiva foi calculado com base na composição das listas de utentes dos médicos participantes, desagregadas por sexo e idade actualizada no início de 2001 e de 2007. É de admitir que a composição da lista de alguns médicos possa não estar perfeitamente actualizada, havendo a tendência para estar sobrestimada. Nestas circunstâncias, as taxas poderão estar subestimadas. Deste modo, as estimativas representadas pelo índice nANTI devem pois ser consideradas como valores mínimos.

Os dados colhidos não permitem fazer qualquer juízo sobre a adequação de cada prescrição, individualmente considerada.

As quinolonas constituíram o terceiro grupo de $\mathrm{AB}$ mais prescrito, praticamente a par com o segundo, os macrólidos, o que revela uma importante preferência por este grupo em MGF. Este padrão de prescrição é, aliás, consistente com a posição que Portugal ocupa entre os estados membros da União Europeia no que respeita às vendas de quinolonas. ${ }^{1}$ Em 2007, a percentagem de prescrição dos $\mathrm{AB}$ deste grupo, na totalidade de $A B$ prescritos na Rede MS, foi de 16,2\% (Quadro IV). Recorde-se que nesse mesmo ano foi estimado para Portugal Continental um valor percentual de consumo para o país de $13,5 \%$, no total de $\mathrm{AB}$ consumidos. ${ }^{3}$ Notese ainda que se, por um lado, o nANTI estimado para este grupo foi menor em 2007 relativamente a 2001, por outro, o valor percentual de prescrição que este grupo representa no total das prescrições aumentou de 2001 para 2007.

A situação face à prescrição de cefalosporinas é mais favorável, tendo-se atingindo em 2007 um valor percentual de prescrição, na totalidade de $\mathrm{AB}$ prescritos, de
$10,1 \%$, praticamente o valor previsto como meta, no Continente, para $2010(10,0 \%){ }^{3}$

É de salientar, contudo, a elevada frequência com que foram prescritas cefalosporinas de terceira geração (26,7\% do total de cefalosporinas).

Refira-se que também foi objecto de notificação o pedido de testes de sensibilidade aos AB (TSA). Apesar de não integrar os objectivos do presente estudo, considera-se pertinente mencionar que a requisição de TSA só ocorreu em 10,6\% das prescrições. Estas requisições concentraram-se fortemente nas doenças do aparelho urinário (em que foram utilizadas em mais de $50 \%$ das prescrições) e, em menor grau, nas doenças dos aparelhos genitais feminino e masculino.

A prescrição de $\mathrm{AB}$ poderia ser aperfeiçoada se aumentasse a percentagem que é apoiada em diagnóstico laboratorial, sempre que este fosse clinicamente indicado, e consequentemente em resultados atempados de TSA, como forma de não só melhorar a eficácia da prescrição, mas também de contribuir para a luta contra o desenvolvimento de resistências aos $\mathrm{AB}$.

Os $\mathrm{AB}$ disponíveis no mercado e as determinantes da sua prescrição modificam-se com rapidez. Justificar-se-á, por isso, que esta temática se mantenha em estudo, utilizando os mesmos métodos de forma a assegurar a comparabilidade dos seus resultados.

Em síntese, na prossecução dos principais objectivos do estudo estimou-se, na Rede Médicos-Sentinela, em 2001 e em 2007:

- O número de $\mathrm{AB}$ prescritos por 1.000 utentes foi menor em 2007 (nANTI 2001 = 93,2 e nANTI 2007 = 81,3);

- O grupo etário dos 0-4 anos apresentou o maior número de prescrições por 1.000 utentes em ambos os anos (233,4 e 143,0 respectivamente); o grupo etário dos 75 e mais anos apresentou um aumento de prescrições por 1.000 utentes de 2001 para 2007 (99,8 e 125,5 respectivamente);

- O número de prescrições de cefalosporinas por 1.000 utentes, assim como a percentagem de prescrição no total de prescrições de $\mathrm{AB}$, foram menores em 2007 (respectivamente, nANTI = 8,2 e 10,1\%) relativamente e a 2001 (respectivamente, nANTI $=11,1 \mathrm{e}$ $11,9 \%)$

- O número de prescrições de quinolonas por 1.000 utentes foi menor em 2007 (nANTI = 13,2) relativamente a $2001($ nANTI $=14,2)$ mas a percentagem de 
prescrição no total de prescrições de $\mathrm{AB}$ foi maior em 2007 (16,2\%) relativamente a 2001 (15,3\%).

Assim, com base nestes resultados pode-se concluir que:

- a percentagem de cefalosporinas prescrita na totalidade de $\mathrm{AB}$ sofreu uma evolução positiva, tendo-se em 2007 atingido praticamente a meta prevista para $2010(10,0 \%) ; 3$

- a percentagem de quinolonas sofreu um aumento ligeiro de 2001 para 2007, apresentando, no entanto, neste último ano um valor que coloca o subgrupo a 5,6 pontos percentuais da meta prevista para 2010 $(10,6 \%){ }^{3}$

De forma a atingir esta meta considera-se importante a promoção de campanhas de sensibilização que tenham como objectivo a diminuição da prescrição deste subgrupo de AB.

\section{AGRADECIMENTOS}

Os autores agradecem aos médicos de Medicina Geral e Familiar da Rede Médicos-Sentinela cuja participação foi indispensável para a realização deste estudo.

\section{REFERÊNCIAS}

1. European Centre for Disease Prevention and Control. European Antibiotic Awareness Day. Summary of Latest European Data on Antibiotic Resistance and Antibiotic Use. ECDC 2009-10-29 . Disponível em: http://www.nvu.nl/uploads/d9/C-/d9C-te07fHVhaUp6Wa0WKA/ Samenvatting-EARSS-2009.pdf [acedido em 25/02/2010].

2. Ministério da Saúde. Direcção-Geral de Saúde. Plano Nacional de Saúde 2004-2010: mais saúde para todos. Lisboa: Direcção-Geral de Saúde; 2004. Vol. I, pág. 78 e Vol. II, pág. 167.

3. Ministério da Saúde.Alto Comissariado para a Saúde. Indicadores e metas do PNS. Disponível em: http://www.acs.min-saude.pt/pns/acessibilidade-ao-medicamento/consumo-de-cefalosporinas-consumo-total-de-antibioticos-em-ambulatorio/ e http://www.acs.min-saude.pt/ pns/acessibilidade-ao-medicamento/consumo-de-quinolonas-consumo-total-de-antibioticos-em-ambulatorio/ [acedido em 30/07/ /2010].

4. Ministério da Saúde. INFARMED. Estatísticas do medicamento 2003. Lisboa: INFARMED - Instituto Nacional da Farmácia e do Medicamento. Direcção de Economia do Medicamento e Produtos de Saúde; 2005. Disponível em: http://www.infarmed.pt/portal/page/portal/ INFARMED/PUBLICACOES/TEMATICOS/ESTATISTICA_MEDICAMENTO/estatistica_2003_0.pdf [acedido em 26/09/2011].
5. Ministério da Saúde. INFARMED. Estatísticas do medicamento 2007. Disponível em: http://www.infarmed.pt/portal/page/portal/INFARMED/PUBLICACOES/TEMATICOS/ESTATISTICA_MEDICAMENTO/EstMed-2007.pdf [acedido em 26/09/2011].

6. Cadieux G, Tamblyn R, Dauphinee D, Libman M. Predictors of inappropriate antibiotic prescribing among primary care physicians. CMAJ 2007; 177 (8): 877-83.

7. Gjelstad S, Dalen I, Lindbaek M. GPs' antibiotic prescription patterns for respiratory tract infections - still room for improvement. Scand J Prim Health Care 2009; 27 (4): 208-15.

8. Observatório Português dos Sistemas de Saúde. O estado da Saúde e a saúde do Estado 2002. Lisboa: Escola Nacional de Saúde Pública; 2002.

9. Palma R. Prescrição de antibióticos no serviço de atendimento complementar. Rev.Port. Clin.Geral 2002 Jan-Fev; 18 (1): 35-52.

10. Branco MJ, Melo M, Nogueira PJ, Martins AP, Falcão, AP. Usos, abusos e mal-usos...utilização de antibióticos. Farm Port 2001; 131: 60-8.

11. Falcão JM, Pisco AM, Simões JA, Falcão IM, Pimenta ZP, Nunes B. Prescrição de antibacterianos em Clínica Geral: um estudo na Rede Médicos-Sentinela. Rev Port Clin Geral 2003 Jul-Ago; 19 (4): 315-29.

12. Direcção Geral dos Cuidados de Saúde Primários. Médicos-Sentinela. Um novo olhar sobre a saúde. Actividades em 1990. Lisboa: DGCSP; 1991.

13. Diário da República, 2 . $^{a}$ série, n. ${ }^{\circ} 252$, de 26 de Outubro de 2004. Despacho n. ${ }^{\circ} 21844 / 2004$, de 12 de Outubro. Classificação Farmacoterapêutica de Medicamentos. Disponível em:http://www.infarmed.pt/ portal/page/portal/INFARMED/LEGISLACAO/LEGISLACAO_FARMACEUTICA_COMPILADA/TITULO_IV/despacho_21884_2004.pdf [acedido em 10/03/2010].

14. World Health Organization. Collaborating Centre for Drug Statistics Methodology, Guidelines for ATC classification and DDD assignment, 2010. Oslo, 2009. Disponível em: http://www.whocc.no/filearchive/publications/2010guidelines.pdf [acedido em 10/03/2010].

15. PASW Statistics 18.0 forWindows. Release 18.0 (23 Aug 2008). SPSS Inc.

\section{DECLARAÇÃO DE INTERESSES:}

Os autores não declaram conflitos de interesse.

\section{ENDEREÇO PARA CORRESPONDÊNCIA}

Eleonora Paixão

Departamento de Epidemiologia

Instituto Nacional de Saúde Dr Ricardo Jorge

Av. Padre Cruz

1649-016 Lisboa

Portugal

Email: eleonora.paixao@insa.min-saude.pt

Recebido em 04/01/2011

Aceite para publicação em 08/03/2012 


\section{ABSTRACT \\ COMPARISON OF ANTIBIOTIC PRESCRIBING IN 2001 AND 2007: A STUDY FROM THE PORTUGUESE SENTINEL NETWORK OF GENERAL PRACTITIONERS}

Objectives: To compare the prescription of cephalosporins and quinolones by general practitioners in the Sentinel Network in 2007 , with the results of a similar study conducted in 2001, determining: the number of antibiotics (AB) prescribed per 1.000 patients and, of all $A B$ prescribed, the proportion of prescriptions of cephalosporines and quinolones.

Design: cross-sectional.

Setting: Portuguese Health Centers belonging to the Portuguese Sentinel Practice Network

Population: Patients registered on the lists of general practitioners belonging to the Sentinel Network

Methods: This study was conducted within the Portuguese Sentinel Network of General Practitioners, allowing for population based estimates to be obtained because the composition of the patient list of the participating physicians is known from the outset. AB prescribing was reported from 2001 and 2007. Annual prescription rates (nANTI = number of prescriptions of antibiotics per 1.000 individuals) by sex and age were calculated.

Results: In 2001, 12.184 prescriptions were studied and in 2007, 9.034 prescriptions for AB were studied. In 2007 the rate of prescription of cephalosporins was 8,2 per 1.000 individuals and the proportion was $10.1 \%$ the total of $A B$ prescribed. This was lower than in 2001 when the nANTI was 11,1 and the proportion was 11,9\%. The rate of prescription of quinolones was lower in 2007 (nANTI = 13,2) than in 2001 (nANTI = 14,2), but the proportion of quinolones among all AB prescriptions was higher in $2007(16,2 \%)$ than in 2001 (15,3\%).

Conclusions: The rate of prescribing of cephalosporins and quinolones per 1.000 individuals was lower in 2007 compared to 2001. The proportion in the total of $A B$ prescribed was lower for cephalosporins, but higher for quinolones in 2007 . Among patients aged 75 years and older the proportion increased in 2007, for both classes (cefalosporines and quinolones), but the increase was statistically significant for quinolones only.

Key-words: Anti-Bacterial Agents; Prescriptions; Therapeutics; Family Physicians; Sentinel Network of General Practitioners. 